\title{
FORMACIÓN UNIVERSITARIA BASADA EN EL DESARROLLO DE COMPETENCIAS PROFESIONALES. APROXIMACIÓN DESDE LA CARRERA DE PSICOLOGÍA DE LA UNIVERSIDAD CATÓLICA DEL URUGUAY ${ }^{1}$
}

\author{
UNDERGRADUATE EDUCATION BASED ON THE DEVELOPMENT \\ OF PROFESSIONAL COMPETENCES. APPROACH FROM THE PSYCHOLOGY \\ DEGREE IN UNIVERSIDAD CATÓLICA DEL URUGUAY
}

González, M. del L.; Silva, M.M. ${ }^{2}$

Universidad Católica del Uruguay, Uruguay

\begin{abstract}
Resumen: En el presente artículo se plantea el debate actual sobre la formación universitaria por competencias. Se recogen algunos autores que han definido el término competencias -entre ellos Peiró, Roe, Barnett- y se realiza un rápido recorrido por las decisiones tomadas en algunas universidades de Europa y América Latina respecto de la formación universitaria en general y la formación de psicólogos en particular. Se dejan planteados los principales desafíos que genera la formación por competencias hoy: revisión del perfil del egresado en diálogo con la sociedad, ajuste de los contenidos, las metodologías y las formas de evaluación de los programas curriculares. Finalmente, se deja planteada la necesaria revisión sobre los conceptos de enseñanza y de aprendizaje que implica la adhesión a la formación por competencias.
\end{abstract}

Palabra clave: Competencia; Formación universitaria, Psicología.

\begin{abstract}
In this paper we discuss the education by competences in higher education. We review the bibliography on the field, guided by authors such as Peiró, Roe, and Barnett. We introduce an overview of the main decisions taken by universities in the United States, Europe and Latin America about undergraduate education in general and psychology education in particular. We state the principal challenges of today's higher education by competences: the professional profile and its relationship with the society demands, curricula tuning, methodology and evaluation of the teaching programs. Finally, we raise the need of reviewing the concepts of teaching and learning in the education by competences.
\end{abstract}

Key Words: Competence, Higher Education, Psychology

\section{INTRODUCCIÓN}

Este artículo recoge material bibliográfico y plantea los principales interrogantes que han surgido en el estudio de las competencias en la formación universitaria en nuestra Facultad. Este tema ha sido prioritario este año en la agenda de "tertulias académicas" que periódicamente se realizan entre los profesores de la Universidad. Además de las lecturas y discusiones, se contó con la visita del Prof. Dr. José María
Peiró, de la Universidad de Valencia (España) reco-nocido experto europeo en el tema.

A continuación se presenta el resultado de una recopilación -de orden conceptualrespecto a la formación universitaria, con especial énfasis en el logro de competencias, recogida de diversos trabajos de destacados académicos.

Se recoge la visión de especialistas de América Latina y de la Unión Europea, quienes plantean una serie de inquietudes respecto a la formación universitaria y su diálogo con las

\footnotetext{
${ }^{1}$ El presente artículo constituye un primer documento de trabajo elaborado a nivel de la Facultad de Psicología, a partir de los contactos iniciales con el tema competencias en la formación universitaria.

CorRespondenciA: Prof.Lic. María Martina Silva, Facultad de Psicología, Universidad Católica del Uruguay, Correo Electrónico: mmsilva@adinet.com.uy
} 
demandas del mercado laboral.

Considerando la importancia de la reflexión en temas relacionados con la formación universitaria en Uruguay, es que ofrecemos un material que tiene la finalidad de acercar al lector a los aspectos centrales de la educación universitaria basada en competencias, que actualmente es motivo de discusión a nivel de políticas educativas.

\section{LA EDUCACIÓN SUPERIOR Y SU COMPLEJIDAD}

La sociedad le exige a las Universidades, respecto a la formación que brindan a los estudiantes, una capacitación que permita un adecuado ejercicio de la profesión.

Ya Morin (2001) presenta las respuestas que la educación debe dar en las diferentes sociedades a los retos de orden científico, tecnológico y del desarrollo humano. Señala, entre otros, que el conocimiento debe ser pertinente y que debe enseñar principios de estrategia que permitan afrontar los riesgos, lo incierto, siempre desde una ética del género humano, que comparta y aplique valores universales.

La educación superior, el conocimiento y la sociedad tienen actualmente una serie de relaciones bidireccionales entre sí. Es difícil comprender plenamente uno de estos elementos desatendiendo los otros. Así, entre estas relaciones podríamos destacar que la educación superior produce su definición de conocimiento, que la sociedad en cierta forma absorbe dando empleo a sus graduados.

A su vez, la sociedad se inclina hoy por formas de conocimiento que tienen un valor de uso en el mercado de trabajo. Por otro lado, la generación de conocimiento a través de la investigación impacta sobre el currículo de la Edu-cación Superior (Barnett., 2001). Se incluye un esquema explicativo de los conceptos analizados por el autor, denominado "triángulo del aprendizaje" (Figura 1).

Un recurso para aliviar las tensiones que a veces se instalan entre educación superior, sociedad y conocimiento que han asumido algunas universidades en los diferentes niveles de titulación es el intento de integrar en el desarrollo de sus prácticas a los colegios profesionales.

Barnett, (2001) reflexiona acerca de la cualidad cambiante que tiene la sociedad y la incidencia de esto en las competencias. Refiere a que esto exige a los profesionales ser agentes de cambio, es decir, no solo deben poder sobrellevar los cambios sino que deben ser capaces de participar activamente en los mismos.

El autor menciona la importancia de estar en constante diálogo con las competencias:

"...No se trata simplemente de poseer "competencias", sino también de tener la capacidad para conversar acerca de ellas, para evaluarlas, para adquirir otras nuevas y descartar las viejas. ...el profesional debe estar capacitado para orientar de nuevas maneras su profesión." (Barnett, 2001:111).

Dentro de este panorama y juego de expec-

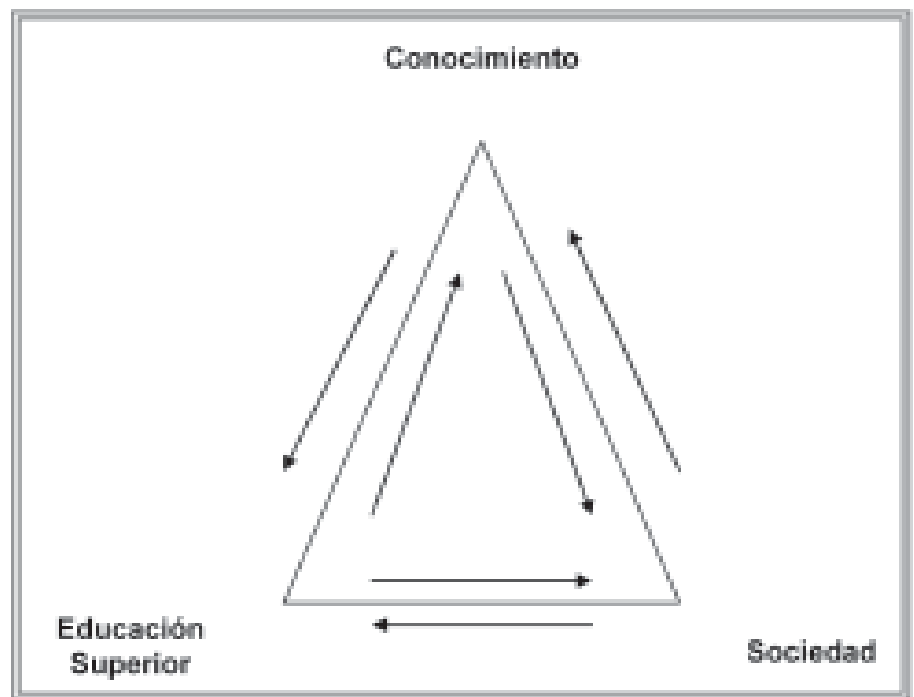

Figura 1: Triángulo de aprendizaje propuesto por Barnett (2001:28) 
tativas, Peña Borrero dice:

"lo que las empresas persiguen detrás de un título universitario no es la garantía de que el candidato está totalmente capacitado para el cargo, sino el respaldo de una formación general y de una disciplina de trabajo con los cuales podrá hacerse frente a los desafíos de la profesión". (en Ocaña, 2003, 16)

Se plantea una crisis a nivel de las universidades ocasionando el comienzo de un proceso de reflexión acerca de lo que enseña y produce. En este marco, el concepto de competencia y la reflexión sobre el currículo universitario ha pasado a ocupar un lugar prioritario en estos últimos tiempos. Se plantea la necesidad de planificar desde el punto de vista del aprendizaje una propuesta de formación competencial.

A nivel de antecedentes, se identifican algunos hitos significativos que han incidido en las transformaciones que la universidad ha tenido en las últimas décadas. Entre ellos, destacamos la Conferencia Mundial de la Educación Superior (UNESCO, 1997), en donde se explicitó la necesidad de actualizar la educación superior a la sociedad del conocimiento y su contribución a elevar de manera efectiva el nivel de vida de la población mundial.

En el marco del proceso de Convergencia Europea de Educación Superior, se asumen estos retos, y recientemente, se ha profundizado y conceptualizado la competencia aplicada al campo de la educación superior, junto con el proceso de integración del crédito académico como criterio rector del diseño curricular universitario.

Asimismo, a partir de la declaración de Bolonia (Ministros Europeos de Educación Superior, 1999, en Yánez, 2008), y en gran medida, apoyados por el proyecto Tuning que reúne más de 100 universidades, se definió un único sistema de transferencia de créditos y la diferenciación entre competencias genéricas y competencias específicas, dando lugar a un movilizador proceso de reforma curricular de las universidades de la región.

Se plantea que las universidades norteamericanas han desarrollado un curriculum nuclear, así como también, aparece definido en el proyecto Tuning europeo la serie de competencias generales, que apuntan al logro de determinados resultados de formación general, de tipo transversal, que se articulan perfectamente con la misión y razón de ser de cada universidad.

\section{APROXIMACIÓN AL CONCEPTO DE COMPETENCIA ${ }^{12}$}

En el proyecto Tuning se define la competencia como:

“... una combinación dinámica de atributos, en relación a conocimientos, habilidades, ac-titudes y responsabilidades, que describen los resultados del aprendizaje de un programa educativo o lo que los alumnos son capaces de demostrar al final de un proceso educativo". (Bezanilla, en Aristimuño, 2004:5)

En el marco del proceso de Convergencia europea de Educación Superior, la concepción de competencias desde la práctica psicológica, es la siguiente:

“...desarrollar y aplicar los principios psicológicos, el conocimiento, los modelos y los métodos en una forma ética y científica con el fin de promover el desarrollo, bienestar, y eficacia en los individuos, grupos, organizaciones y sociedad..." (Peiró, 2008)

Por otra parte, Perrenoud (en Aristimuño, 2004:3) propone la siguiente definición:

"capacidad de actuar de manera eficaz en un tipo definido de situación, capacidad que se apoya en conocimientos, pero no se reduce a ellos".

En este sentido, Aristimuño agrega que esto plantea un problema latente para la educación superior, ya que el pasaje de la adquisición de un conjunto de conocimientos al dominio de una competencia no es algo lineal, y mayoritariamente los docentes universitarios poco conocen este proceso.

Desde la perspectiva de Barnett (2001:108):

"decir que un individuo es competente, significa afirmar que sus acciones alcanzan cierto estándar". Cuanto más alto sea el nivel de competencias, más exigentes serán los estándares esperados.

Roé (2003:4) explica en su artículo que si bien se ha conceptualizado de manera diferente la noción de competencia, existe un creciente consenso que alienta la siguiente definición: "...una capacidad aprendida para realizar adecuadamente una tarea, funciones o rol".

\footnotetext{
${ }^{\prime}$ En el presente trabajo se presenta algunas de las concepciones acerca de competencias, un concepto sobre el que existen varias perspectivas, sin embargo, no se hará un análisis de las diferentes conceptualizaciones que existen al respecto, ya que no es objeto de este artículo.
} 
Asimismo plantea que las competencias presentan dos rasgos particulares, el primero, es que se relaciona con un tipo específico de trabajo para ser realizado en un contexto particular, y el segundo, es que integra diversos tipos de conocimientos, habilidades y actitudes. Estos últimos son típicamente adquiridos durante el proceso educativo, a través de sucesivas situaciones de aprendizaje escolar. Destaca que las competencias se desarrollan en un proceso de "aprender haciendo" en la situación concreta de trabajo, durante las prácticas externas o por medio de situaciones controladas de aprendizaje basada en simulación. Aclara también que los conocimientos, habilidades y actitudes son más elementales que las competencias, ya que estas, pueden ser desarrolladas aisladamente, evaluadas por separado, y aplicadas a varias competencias.

Roé (2003) propone un modelo arquitectónico sobre competencia, similar a un templo griego, en donde se especifica los distintos elementos que se deben considerar para cualquier ocupación; destaca que los ingredientes a partir de los cuales se construye las competencias, son las subcompetencias, así como, los conocimientos, habilidades y actitudes.

Se entiende por subcompetencia, aquella competencia genérica que no es suficiente para la realización de cualquier trabajo en una organización, pero si aparece en combinación con otras cualidades. Por ejemplo, una subcompetencia es usar un software de procesamiento de textos, administrar test, buscar documentación, saber entrevistar, entre otras.

Por conocimientos, se considera al conjunto de información teórica y práctica producido en los diferentes campos de la psicología. Con respecto a las habilidades, se incluye por ejemplo, la comunicación oral y escrita, análisis de problemas, observación, colaboración en equipo, entre otras. $Y$ finalmente, las actitudes, se relacionan con la precisión, el respeto por el otro, apertura hacia la crítica, orientación al servicio, compromiso, integridad, etc.

Asimismo aclara en su artículo sobre el tema, que no debe confundirse las competencias con las aptitudes, rasgos de personalidad u otras características de la persona, que son disposiciones más estables y conforman la base por la que los individuos aprenden los conocimientos, habilidades y actitudes, así como las sub-competencias y competencias.

Por otra parte, plantea que la competencia es necesaria pero no suficiente para alcanzar una adecuada ejecución. En este sentido, considera que una persona que es considerada competente para desempeñarse en determinada tarea, puede no siempre ejecutarla bien, debido a factores como la falta de motivación, fatiga, enfermedad, etc.

A continuación agregamos el diseño del modelo de competencia propuesto por Roé (2003) que permite visualizar el concepto y sus dimensiones (Figura 2).

\section{TENDENCIAS EN LA FORMACIÓN ACADÉMICA EN AMÉRICA LATINA}

Tobon (2007) de la Universidad de Valparaíso, plantea que el enfoque de competencias asume los retos no resueltos de la educación tradicional, asumiendo los desafíos de definir currículos pertinentes, la implementación de estrategias explícitas para desarrollar la idoneidad, el logro de una adecuada integración entre la teoría y la práctica. Todo esto, apostando a una formación integral, siguiendo a Delors (1997) con el aprender a ser-hacer-conocer y convivir en su propuesta de educación de cara al siglo XXI. Además, agrega los nuevos retos que se suman a los mencionados, como por ejemplo, las certificaciones parciales para facilitar la empleabilidad, el ensamblaje entre la formación de pregrado-postgrado-educación continua, y el alcance de la certificación de la idoneidad.

Asimismo, destaca en su trabajo, algunos países de América Latina que se encuentran en proceso de evaluación de sus propuestas académicas a nivel terciario, entre los cuales menciona:

I. México: Se está trabajando en el desarrollo de un modelo educativo por competencias en la formación del estudiante universitario.

II. Chile: Se puede identificar la existencia de avances significativos en el abordaje del diseño del currículum.

III. Argentina: Se habla de integración de niveles y la elaboración de una nueva propuesta curricular a nivel universitario.

IV. Brasil: Al igual que en Chile, se está abordando el diseño del currículum basado en competencias.

V. Perú: Se menciona el trabajo de evaluación que se está realizando del programa académico con el objetivo de poder abordar los cambios desde una perspectiva de competencias. 


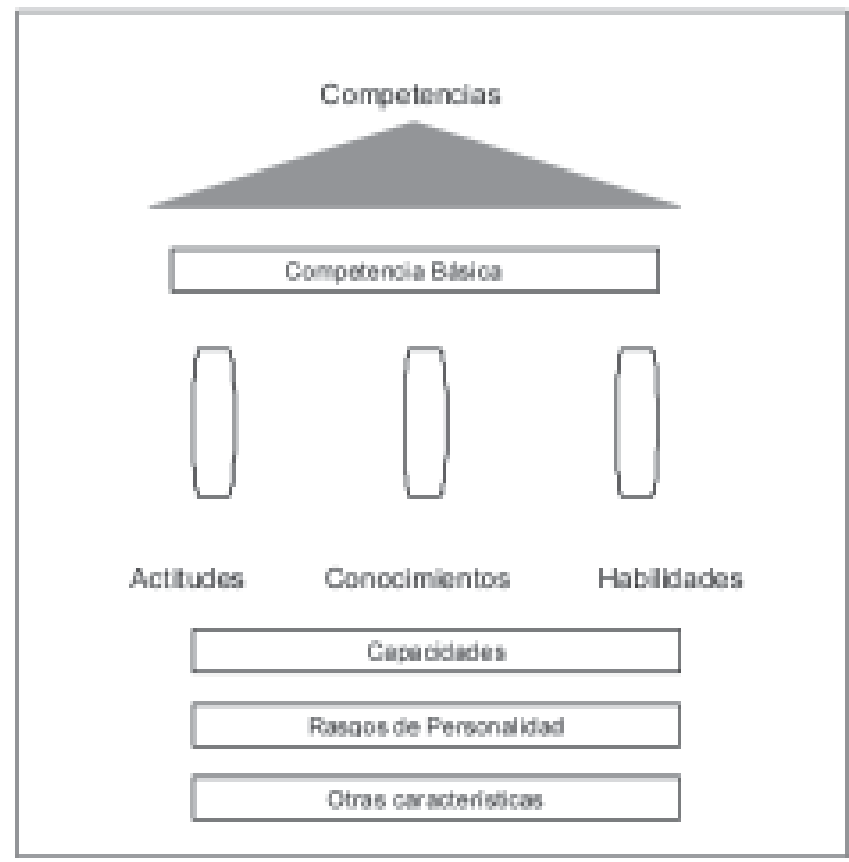

Figura 2 : Modelo do Compotenclas propuesto por Ros (2003)

\section{ANTECEDENTES DE LA UNIVERSIDAD CATÓLICA DEL URUGUAY}

En el 2003, la Vicerrectoría Académica de la Universidad Católica, planteó una serie de objetivos destinados a guiar el proceso de reforma curricular. Aristimuño (2004:5-6) destaca algunos de los objetivos propuestos en ese entonces:

- "Avanzar hacia la educación por competencias y la educación integral."

- Introducir progresivamente dos o tres "núcleos curriculares", entendidos como "paquetes" de asignaturas transversales, a ser tomadas por estudiantes de diversas carreras (o eventualmente, de todas las carreras de la Universidad).

- Avanzar hacia un sistema de créditos que combine materias transversales, materias especificas obligatorias y materias electivas (normalmente a ser elegidas entre las materias obligatorias de otras carreras).

- Innovar en métodos de evaluación.

- Introducir un conjunto de asignaturas específicamente orientadas al logro de competencias generales y transversales".

Otro de los aspectos abordados fue comenzar a definir el perfil del graduado de cada carrera, incluyendo las competencias que se pretenden desarrollar y lograr alcanzar al finalizar el proceso educativo. En este sentido, se alude a la idea de que existen competencias también a ser logradas a lo largo del proceso, y no solamente al concluir el mismo.

Las competencias genéricas, definidas por la Universidad Católica en dicha instancia, en coherencia con la

Misión y con los conceptos de educación general y currículo nuclear desarrollados fueron (Aristimuño, 2004:6):

1. "Competencias comunicativas fluidas y de calidad, en el lenguaje oral y el escrito.

2. Gestión y organización de personas y recursos materiales.

3. Aprendizaje permanente, meta-cognición y competencias heurísticas.

4. Innovación, iniciativa y emprendimiento.

5. Discernimiento espiritual.

6. Intervención competente en situaciones que demandan mayores niveles de humanización".

Desde la perspectiva de Roé (2002):

"el enfoque de las competencias modifica los puntos de vista convencionales sobre la forma de aprender y de enseñar, pues el aspecto central, como vimos, no es la acumulación pri- 
maria de conocimientos, sino el desarrollo de las posibilidades que posee cualquier individuo, mediante fórmulas de saber y de saber hacer contextualizadas".(en Aristimuño, 2004:7)

La autora sostiene que la piedra angular del diseño curricular es la definición de las competencias que se pretenden lograr; para ello, se sugiere la revisión del perfil de egresado. Asimismo, se plantea la importancia de la articulación entre las competencias definidas como transversales o generales y aquellas que desarrolla una asignatura concreta, así como también, la importancia de considerar a partir de esas competencias (coherentes con las de mayor nivel de generalidad) el tipo de contenido, metodologías y formas de evaluación que mejor se ajusten a los objetivos de la asignatura.

El enfoque de competencias ha despertado resistencias especialmente entre los docentes universitarios, ya que muchas veces se le asocia a una mentalidad instrumental que responde a las presiones de la sociedad. En experiencias mencionadas en este artículo se trata de acompañar estos cambios propiciando la reflexión continua sobre el diseño curricular, las prácticas de enseñanza en el aula y las formas de evaluación.

El desafío central del enfoque por competencias, consiste en no circunscribir su logro a asignaturas determinadas, sino que cada docente, teniendo claro en su horizonte el perfil del egresado que se quiere lograr, repase sus prácticas en sentido amplio, interrogándose sobre las competencias generales y específicas que está estimulando en sus estudiantes.

En este sentido, el centro de atención para instrumentar los cambios curriculares debe necesariamente trasladarse de la enseñanza del docente a los aprendizajes que logran los alumnos. (Aristimuño, 2004). Las nuevas definiciones de crédito educativo, centrado en el trabajo que el estudiante debe realizar para superar las exigencias de una materia, más que en función de las horas docentes que reciba, constituye un buen ejemplo de lo que acabamos de decir.

Este cambio en el centro de atención requiere así de otro perfil docente, que debería transformarse en supervisor permanente de los aprendizajes de los alumnos.

PERSPECTIVA EUROPEA SOBRE LA FORMACIÓN DEL PSICÓLOGO
Según Barnett (2001:112): "cuando hacemos una afirmación respecto de las competencias, también la estamos haciendo respecto del currículo que deseamos...las implicaciones de la definición de competencias en el currículo es inevitable".

En el marco del proceso de Convergencia europea de Educación Superior, la concepción de competencias desde la práctica psicológica, es la siguiente:

“... desarrollar y aplicar los principios psicológicos, el conocimiento, los modelos y los métodos en una forma ética y científica con el fin de promover el desarrollo, bienestar, y eficacia en los individuos, grupos, organizaciones y sociedad..." (Peiró, 2008).

Asimismo, Peiró (2008) destaca en el marco de esta conceptualización, el desafío de lograr definir y analizar las principales competencias para el ejercicio profesional como psicólogo en los diferentes ámbitos de intervención.

A continuación agregamos el diseño del modelo de competencia propuesto por Roe (2003), empleado por el estudio europeo y que permite visualizar el concepto y sus dimensiones, en la Figura 3.

\section{COMPETENCIAS DE LOS PSICÓLOGOS PARA EL EJERCICIO PROFESIONAL}

Siguiendo con los aportes de Roe sobre este tema, plantea que es posible elaborar un perfil de competencias del psicólogo, en el cual cada uno de los elementos descriptos deben ser definidos en términos de contenidos y niveles. Según el Sistema Integrado de Perfilado (Roe, 2001), se debe seguir los siguientes pasos para desarrollar un perfil de competencia de cualquier ocupación:

1. Análisis ocupacional: implica reunir la información acerca del rol, funciones y tareas que debe cumplir en un trabajo específico.

2. Análisis de competencias: se incluye las competencias requeridas, junto con los conocimientos, habilidades y actitudes necesarias, así como, las disposiciones subyacentes, como las capacidades, rasgos de personalidad, y otras carac-terísticas.

3. Modelizar las competencias: ejemplificar un modelo que muestre las relaciones entre las competencias y los conocimientos, habilidades y actitude más importantes.

4. Contrastar el modelo de competencias: evaluar la validez del modelo y definir los in- 


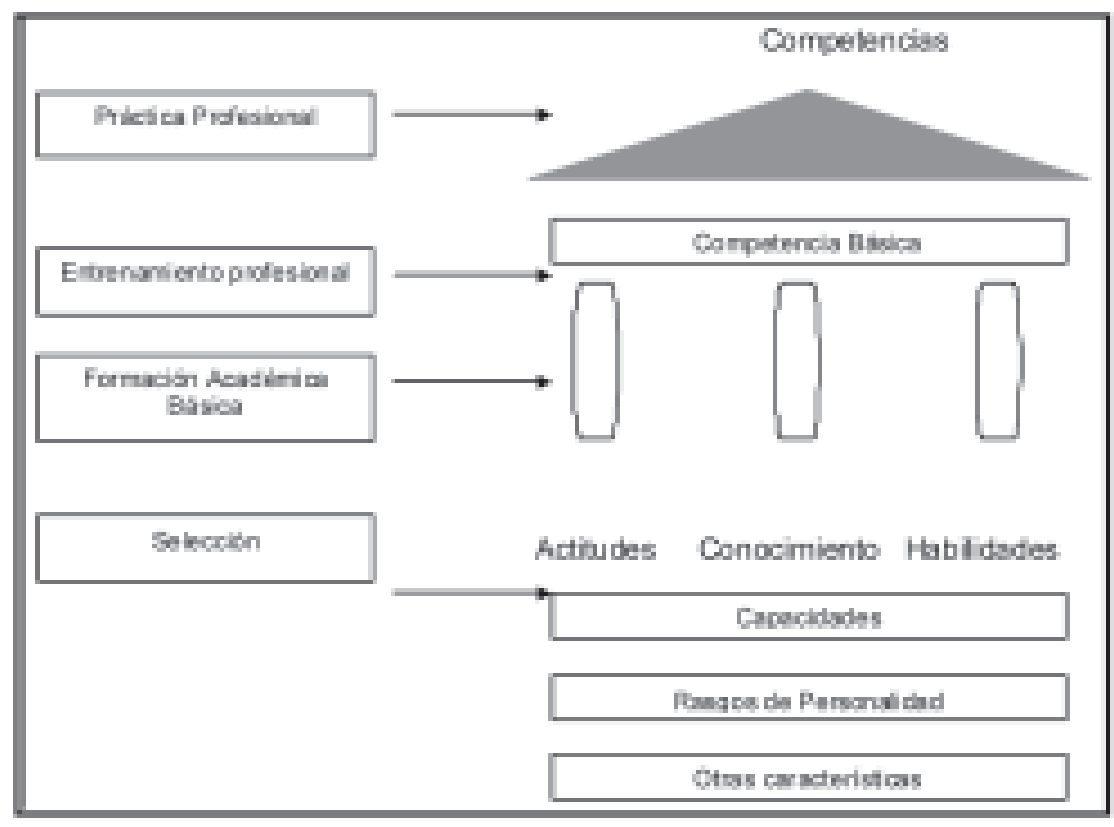

Finura 3

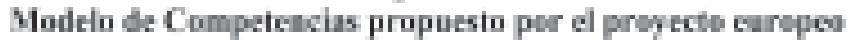

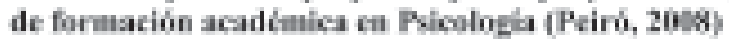

dicadores que contribuyen o que predicen las competencias.

Por otra parte, el proyecto europeo de formación académica en psicología, reconoce la existencia de dos fuentes para el diseño de un currículo de formación que se ajuste al modelo de competencias propuesto anteriormente:

A. Input Información que se deriva de la disciplina científica, es decir, la formación en base a las materias curriculares que deben estar contenidas en el plan de estudio.

$B$. Output Información que se deriva del desempeño competente del ejercicio profesional en el nivel de inicio de la práctica autónoma.

Asimismo, se considera que las competencias del profesional en psicología deben ser en función de los diferentes roles profesionales: Psicología de las Organizaciones y el Trabajo, Psicología Clínica y Salud, Psicología Educativa, Psicología Social y Comunitaria.

En cuanto a la Facultad de Psicología de la Universidad Católica del Uruguay, continúa apostando a una formación actualizada y continua, define un perfil de egresado que promueve la salud mental y el mejoramiento de la calidad de vida en los diferentes ámbitos de la sociedad. Se educa desde un enfoque de prevención y con capacidad para el diagnóstico y posterior tratamiento de los problemas psicológicos. La propuesta académica se apoya en una formación plural y sólida, con conocimiento de investigación en psicología, la cual brinda herramientas suficientes para el futuro ejercicio ético de la profesión. Asimismo, se articulan a lo largo de la carrera diversas prácticas supervisadas, que habilitan el aprendizaje del ejercicio profesional competente en área clínica, laboral y educacional.

Los cambios de enfoque acerca de la concepción sobre cómo educar y los criterios que definen una propuesta académica basada en el desarrollo de competencias profesionales, influyen en las nuevas perspectivas que las universidades están adoptando. Obviamente esto no es ajeno a la Universidad Católica, específicamente, a la Facultad de Psicología, la cual, como hemos informado, en este último tiempo ha ingresado en un proceso de análisis interno acerca de los distintos aspectos que la definen como centro de formación profesional.

Los aportes de los autores citados en este breve recorrido bibliográfico, dan cuenta de un proceso complejo y continuo que involucra a todos los agentes de la educación. Se evidencia, a nivel de la educación superior, la necesaria armonía que debe existir entre los contenidos 
a presentar, las metodologías de enseñanza, y el profesional que se quiere formar, exigiendo continuas instancias de reflexión con la comunidad académica.

\section{REFERENCIAS}

Acosta, H. (2006). El modelo educativo por competencias centrado en el aprendizaje y sus implicaciones en la formación integral del estudiante universitario. Consultado en mayo, 25, 2008 en www.congresoretosyexpectativas.udg.mx.

Aristimuño, A (2004): Las competencias en la educación superior, ¿demonio u oportunidad?. Ponencia presentada en el Tercer Congreso Internacional Docencia Universitaria e Innovación (CIDUI), organizado por las Universidades de Gerona, Politécnica de Catalunya, de Barcelona y Autónoma de Barcelona, Gerona, mes de julio.

Aristimuño, A, González, M. L. y Luján, C. (2001): Organización y docencia universitaria: un diálogo posible en: Gairín, J. y Fernández A. (Dirs): Planificación y gestión en instituciones de formación. Barcelona: Praxis Vol 1. 75-100

Barnett, R. (2001). Los límites de las competencias. El conocimiento, la educación superior y la sociedad. Barcelona: Gedisa

Delors, J. et. al (1997). La educación encierra un tesoro. México: UNESCO.

Morin, E. (2001). Los siete saberes necesarios para la educación del futuro. Barcelona: Paídos lbérica, S.A.

Ocaña A. (2003). Formación General en la Univer- sidad. Presentado en: Conferencia XXIV-A del Seminario General del Simposio Permanente sobre la Universidad. Montevideo, Uruguay.

Peiro, J (2003). La enseñanza de la Psicología en Europa. Un proyecto de titulación Europea. Papeles del Psicólogo, 24 (086), 25-33.

Peiro, J. (2008). Curriculum universitario basado en competencias: una propuesta europea. Conferencia brindada en el marco de las Tertulias Académicas. Montevideo: Facultad de Psicología, Universidad Católica del Uruguay. En agosto, 13, 2008.

Posada Álvarez, R. (2003). Formación Superior basada en competencias, Interdisciplinariedad y trabajo autónomo del estudiante. Consultado en mayo, 16, 2008 en www.rieoei.org/ deloslectores/648Posada.PDF

Roe, R. (2003). ¿Qué hace competente a un psicólogo?. Revista Cientifica de America Latina y el Caribe, España y Portugal, 24: 14-23

Sánchez González, J. (2003). La formación del Psicólogo en una perspectiva Internacional. Una breve selección documental comparada. Consultado en agosto, 30, 2008 en www.icfes.gov.co/cont4/ ecaes/docs

Tobon, S. (2007). Aspectos generales del enfoque de las competencias. Consultado en mayo, 17, 2008 en www.tecnologicocomfacauca.edu.com

Yánez, C. (2008). Las competencias en el curriculo universitario: implicaciones para diseñar el aprendizaje y para la formación del profesorado. Consultado en mayo, 4, 2008 en www.redu. um.es/Red_U/m1/ 